\title{
Serial transverse enteroplasty and nipple valve construction, two life saving techniques for patients with short bowel syndrome, a report of 5 cases
}

\author{
Mojtaba Shafiekhani ${ }^{1,2}$, Nazanin Azadeh ${ }^{1}$, Kiarash Ashrafzadeh ${ }^{1}$, Maryam Esmaeili ${ }^{1}$ and Hamed Nikoupour ${ }^{{ }^{*}}$
}

\begin{abstract}
Background: Various abdominal pathologies end up with surgical resection of small intestine. When the small intestine remnant is too short for adequate fluid and micronutrients absorption, short bowel syndrome is diagnosed. The disabling condition needs a multidisciplinary approach to design parenteral nutrition, care for thrombotic, hepatic and infectious complications and gradually wean the patient from parenteral nutrition. Various surgical techniques have been introduced to increase absorptive mucosa and enhance the intestinal adaptation process. Serial transverse enteroplasty and nipple valve reconstruction are among the procedures, which will be discussed in the current article.

Case presentation: Herein, we presented 5 cases of short bowel syndrome as a consequence of abdominal laparotomies, patients were referred to our center to receive parenteral nutrition and to be prepared for the final autologous gastrointestinal reconstruction or intestinal transplantation, if indicated.

Conclusion: Patient's age, performance status and bowel remnant length determines the appropriate technique for autologous gastrointestinal reconstruction. Serial transverse enteroplasty is designed to increase bowel's length by creating zigzag patterns through dilated bowel loops. Presence of ileocecal valve is crucial to delay intestinal transit time and to prevent colonic bacterial transfer to ileum. Patient's with ileocecal valve loss benefit from creating an artificial valve, namely, nipple valve.
\end{abstract}

Keywords: Autologous gastrointestinal reconstruction, Case report, Enterocutaneous fistula, Intestinal failure, Serial transverse enteroplasty, Short bowel syndrome

\section{Background}

Intestinal failure (IF) is a state of reduced effective bowel surface to meet the minimum necessary body demands of macronutrients, electrolytes and fluids, so that intravenous fluid resuscitation or parenteral nutrition (PN) is inevitable to maintain health and prevent malnutrition [1]. Diminished bowel's function is

\footnotetext{
*Correspondence: nikoupour@gmail.com

1 Shiraz Transplant Research Center, Shiraz University of Medical Sciences, Shiraz, Iran

Full list of author information is available at the end of the article
}

the consequence of an anatomic or functional loss of absorptive mucosa. In adults, the most common causes of short bowel syndrome (SBS) and consequent IF are complications of abdominal surgery due to catastrophic intestinal insults caused by trauma, volvulus, mesenteric ischemia, malignancies, enterocutaneous fistulae and extensive bowel resection due to Crohn's disease [2]. Moreover, substantial diseased gut's absorptive mucosa may result in functional SBS, the condition is seen in inflammatory bowel disease, radiation-induced enteritis and other enteropathies [3]. IF is a debilitating 
condition with various complications including liver steatosis, osteopenia, renal stones, dehydration and malnutrition, lowering the patients' quality of life and survival [4]. After an extensive loss of bowel's mucosa, the intestine undergoes adaptive changes to increase the absorptive surface. The changes include increased villi height and crypt depth and dilatation of the remaining intestinal segments [5]. As the adaptation process goes on, gradually the degree of diarrhea and fluid loss decreases and the patient might need less frequent or no PN. The process may continue for years [6]. Oral or enteral feeding stimulates intestinal adaptation; therefore, surgeons are widely encouraged to start feeding as soon as possible after bowel resection [7]. However, adaptive changes are not always desirable, as bowel dilatation may lead to bacterial overgrowth and dysmotility [8]. Acutely ill and metabolically unstable IF patients who have survived a complicated abdominal surgery are cared for in intestinal rehabilitation units (IRU) by a multidisciplinary team (MDT) including surgeons, pharmacists, nutritionists and various other specialists [9]. The MDT designs essential PN and hydration, manages fistulae, open abdomen, PN complications and infections until the patient becomes independent of inpatient $\mathrm{PN}$ or is prepared for further abdominal operations including autologous intestinal reconstruction (AGIR) or intestinal transplantation [10-12]. AGIR refers to various surgical techniques to enhance intestinal absorptive mucosa in order to minimize dysmotility and the need for intestinal transplantation [13]. Although still debated, AGIR is reserved for patients who despite intensive medical efforts, are not able to attain a PN-free life [8]. Serial transvers Enteroplasty (STEP) is a relatively new AGIR technique with 15 years of experience. The technique is designed to lengthen the intestine by creating zigzag lumens in dilated bowel segments [14]. In previous studies, STEP procedure has shown promising results as an ultimate technique to manage SBS. Various studies have reported $20-60 \%$ bowel lengthening by performing STEP, as well as an approximate $43 \%$ successful rate to wean the patients from PN $[15,16]$.

Ileocecal valve (ICV) plays a notable role in delaying intestinal transit time and preventing colonic bacterial transfer to the small intestine. Patients with ICV loss benefit from artificial valve reconstructions. Nipple valve is created by intussuscepting small intestine to colon. The valve delays distal intestinal motility, subsequently reserves more time for absorption [17].

In this report, we briefly review the history and management of 5 patients with SBS and IF, for whom STEP or nipple valve creation was done in the AbuAli Sina Organ Transplnat Hospital, Shiraz, Iran. The hospital is the unique referral center for IF patients in Middle East.

\section{Case presentation \\ Case 1 \\ History and presentation}

A 58-year-old male patient was admitted with mesenteric ischemia in a local hospital. The patient had immediately undergone open laparotomy, assuming that the remaining bowel was too short in length (Fig. 1A), the surgeon had decided to close the abdomen without a certain intervention.

\section{Management}

The next day, our team was called and attended the patient for laparotomy. The gangrened bowel sections were resected and the viable bowel remnant measured $35 \mathrm{~cm}$ distal to ligament of Treitz, as well as $5 \mathrm{~cm}$ of remaining ileum (Fig. 1B). A gastroduodenostomy tube was inserted and the patient was transferred to IRU, where he received PN for 8 weeks. At the end of this period, a radiologic contrast study revealed dilatation of the proximal section of the bowel, for which he was scheduled to undergo an AGIR operation. During the recovery period, the length of the bowel had increased to $55 \mathrm{~cm}$ (Fig. 1C), which was further increased to $75 \mathrm{~cm}$ with STEP procedure. Considering the $5 \mathrm{~cm}$ ileal remnant, the final length of the remaining bowel was $80 \mathrm{~cm}$ at the end of the procedure (Fig. 1D).

\section{Outcome}

By preserving bowel's continuity and patient's tolerance of oral nutrition, he was discharged from the hospital 10 days later to the last surgery. On the time of preparing the article (22 months following discharge date) the patient had no complaint or no need to be admitted in the hospital due to IF symptoms and signs.

\section{Case 2}

\section{History and presentation}

In a local hospital, a 47-year-old male patient had undergone open laparotomy and resection of gangrened bowel and anastomosis of viable bowel segments due to mesenteric ischemia. At the end of the procedure, the remaining bowel measured $20 \mathrm{~cm}$ including left colon (Fig. 2). The patient was then referred to our center.

\section{Management}

A radiologic contrast study was done which revealed the bowel diameter to be $5 \mathrm{~cm}$; therefore, the patient was a candidate for the STEP procedure. While exploring the abdomen in the OR, we estimated the remaining bowel's length to be $20 \mathrm{~cm}$. We performed STEP for duodenum 


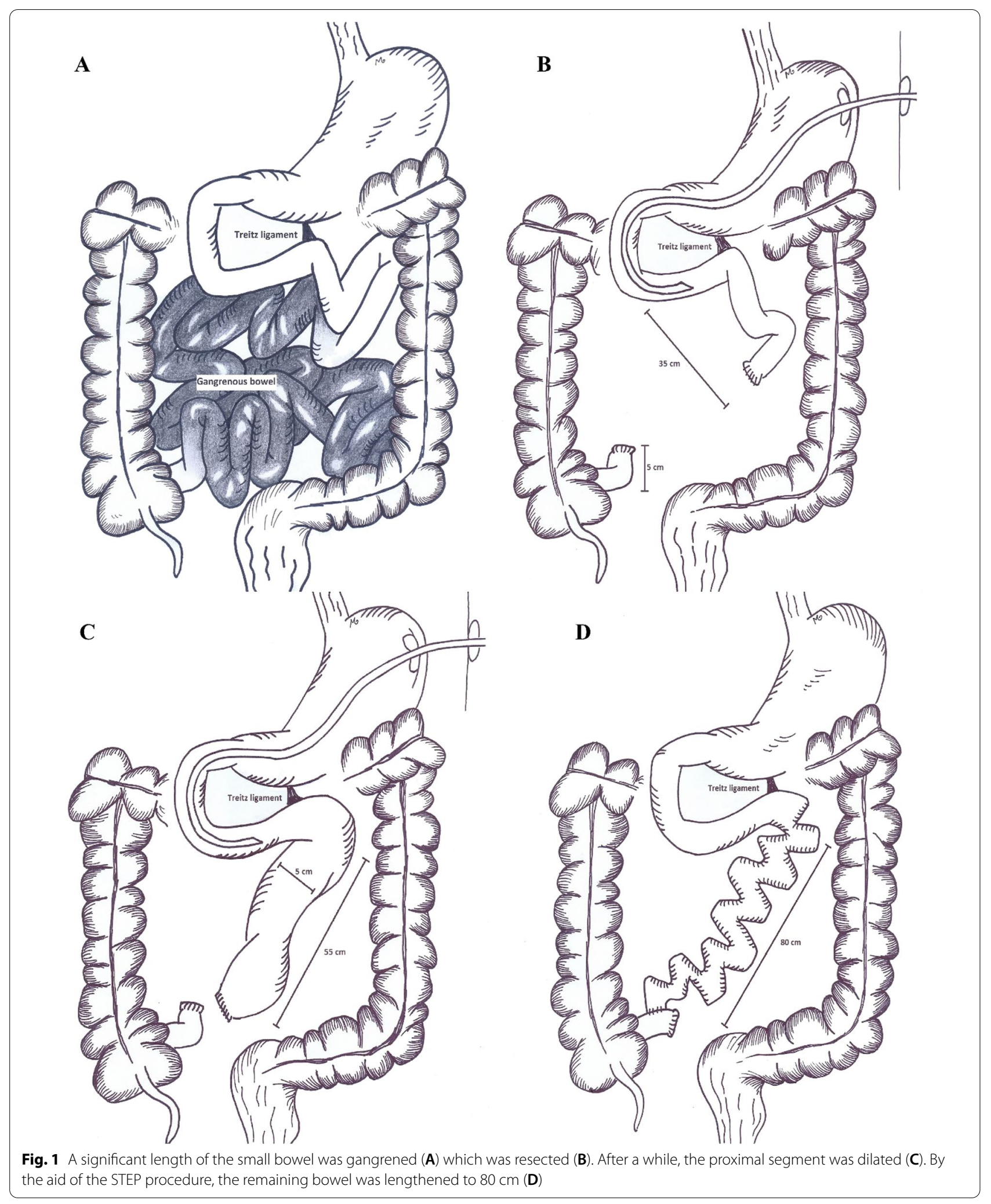




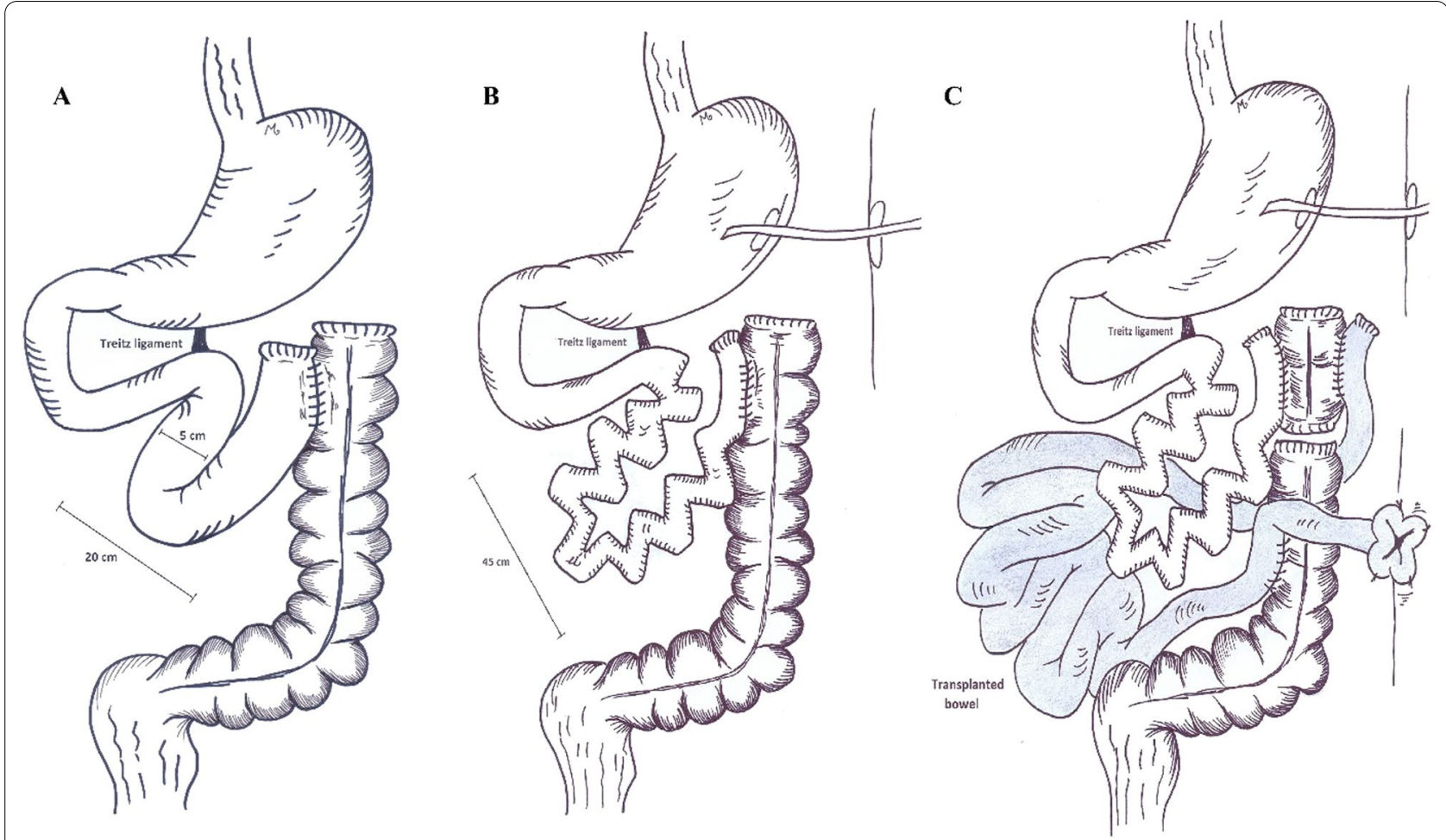

Fig. 2 The patient was referred after extensive resection of small bowel (A), with the aid of the STEP procedure, the bowel's length was increased to $45 \mathrm{~cm}(\mathbf{B})$, due to failure of AGIR, a small intestine transplantation was done (C)

and the $20 \mathrm{~cm}$ long remnant bowel. The bowel's length was increased to $45 \mathrm{~cm}$ with the procedure. Afterwards, the patient received PN for 1 month and was dismissed from IRU. During the upcoming months, he was frequently admitted with dehydration. Consequently, he was put on the waiting list for small intestinal transplantation.

\section{Outcome}

Eventually, he underwent isolated small intestinal transplantation, which was uneventful for next 6 months. At the end of the 6th month, the patient was admitted with fungal infection of the transplanted organ, which led to sepsis and death.

\section{Case 3}

\section{History and presentation}

A 36-year old lady was transferred to our center with internal bowel herniation and in an unstable hemodynamic state. Patient's past medical history included liver transplantation due to primary sclerosing cholangitis with Roux-en-Y common hepatic duct anastomosis, 4 years prior to the current admission. She had no history of concurrent inflammatory bowel disease.

\section{Management}

She was immediately transferred to OR for an emergent laparotomy. The gangrened bowel segments were resected and the hepaticojejunostomy was taken down. With the aid of Cattell-Braasch maneuver and in a retro-colic position, the bile duct was anastomosed to the ileum. The proximal of the remaining bowel which had a diameter of $6 \mathrm{~cm}$ was closed and a gastroduodenostomy tube was inserted (Fig. 3). At the end of the procedure, the remnant of bowel measured $35 \mathrm{~cm}$ from ligament of Treitz to the jejunojejunostomy anastomosis site and $50 \mathrm{~cm}$ from anastomosis site to the ileocecal valve. The patient was then transferred to the intensive care unit, where the immunosuppressive medications (Tacrolimus, Prednisolone) were continued. A week later, in a stable hemodynamic state, she underwent another laparotomy to lengthen the intestinal remnant. The STEP procedure was done on the proximal segment of the bowel, increasing its length from 35 to $50 \mathrm{~cm}$. Furthermore, the segment was anastomosed to the highest possible side of the Roux limb. 

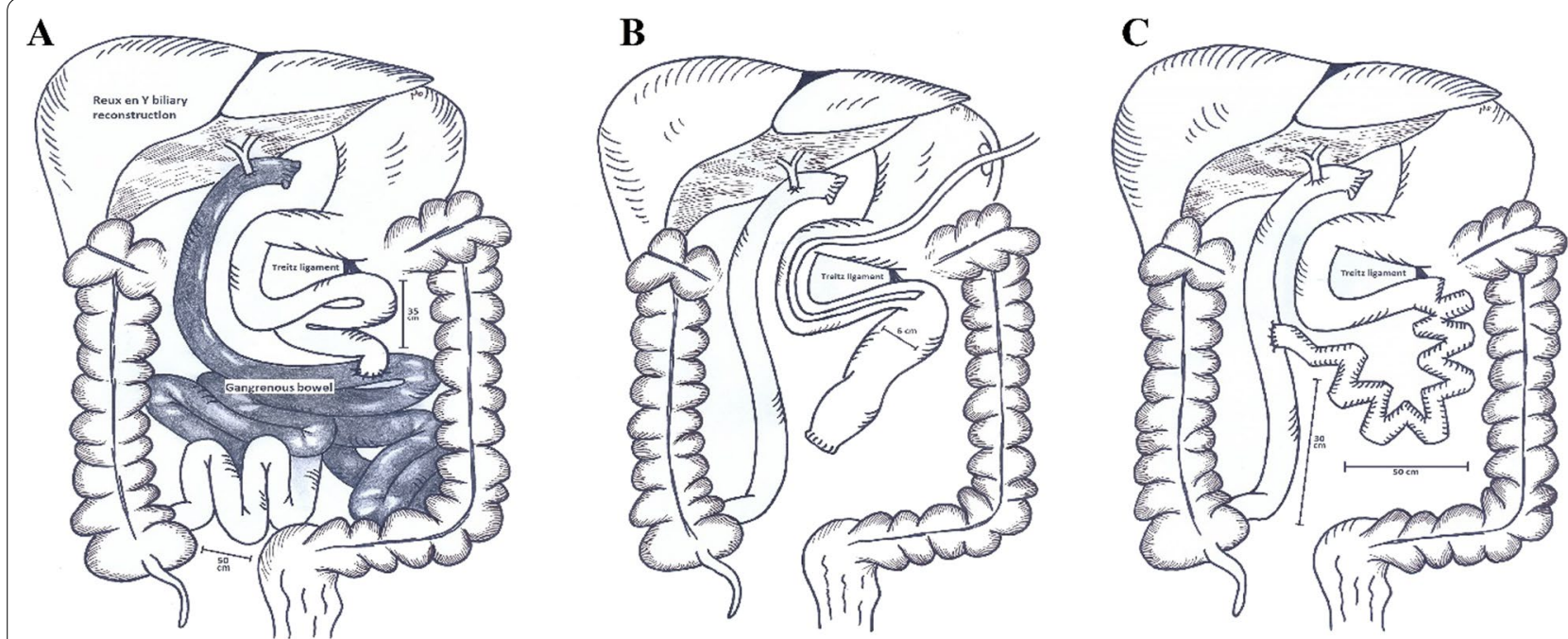

Fig. 3 The liver recipient was admitted with internal bowel herniation and consequent bowel gangrene (A), the gangrened segments were immediately resected, the bile duct was anastomosed to ileum and a gastroduodenostomy tube was inserted (B). A week later, STEP was done on dilated remaining bowel $(\mathbf{C})$

\section{Outcome}

On the 5th post-operation day, oral nutrition was started for the patient and 5 days later, she was dismissed from the hospital. In the most recent follow up, after 15 months of being discharged from hospital we assessed that the adaptation process helped the patient have a relative acceptable quality of life.

\section{Case 4}

\section{History and presentation}

In a local hospital, a 71-year old diabetic patient had undergone resection and anastomosis of gangrened small intestine segments due to mesenteric ischemia. The patient was later referred to our center with enterocutaneous fistula.

\section{Management}

During the next month, we managed the open abdomen and the patient received $\mathrm{PN}$ while instructed with nil per os (NPO) order. On the 35th day, the fistula had already been closed and in addition to PN, oral nutrition was started for him. Eight weeks after the primary operation, the patient was scheduled for AGIR. During the abdominal exploration, the length of the bowel remnant to the fistula was estimated to be $25 \mathrm{~cm}$. After resection, $20 \mathrm{~cm}$ of bowel and the descending colon were the remaining segments (Fig. 4). Considering the fact that the patient was not an ideal candidate for small intestine transplantation and he would be dependent on daily $\mathrm{PN}$, we decided to create a nipple valve by intussuscepting the small intestine to colon. Therefore, bowel's continuity was preserved. Additionally, a gastrostomy tube was inserted as a vent.

\section{Outcome}

On the 5th post-operation day, the patient tolerated oral nutrition. During the next month, PN was gradually tapered to the lowest necessary frequency and he was discharged from the hospital with a weekly need for PN and after 2 months the patient needed no more PN. Later, patient was admitted with dehydration and severe diarrhea which was managed by fluid resuscitation and high dose loperamide for 1 month. During the past 6 months and until the latest follow up, he has gained weight and complains of no more diarrhea with $2 \mathrm{mg}$ of Loperamide, administered three times in the day.

\section{Case 5}

\section{History and presentation}

A-52-year old lady was admitted in a local hospital with small bowel ischemia and had undergone resection of the gangrened bowel segments with creation of an ostomy. She was then transferred to our IRU to receive PN.

\section{Management}

After 8 weeks of ostoma care and receiving PN as well as regular oral nutrition, she was scheduled for AGIR. A nipple valve was created in the $43 \mathrm{~cm}$ long remaining bowel and a gastrostomy was inserted as a vent (Fig. 5). 

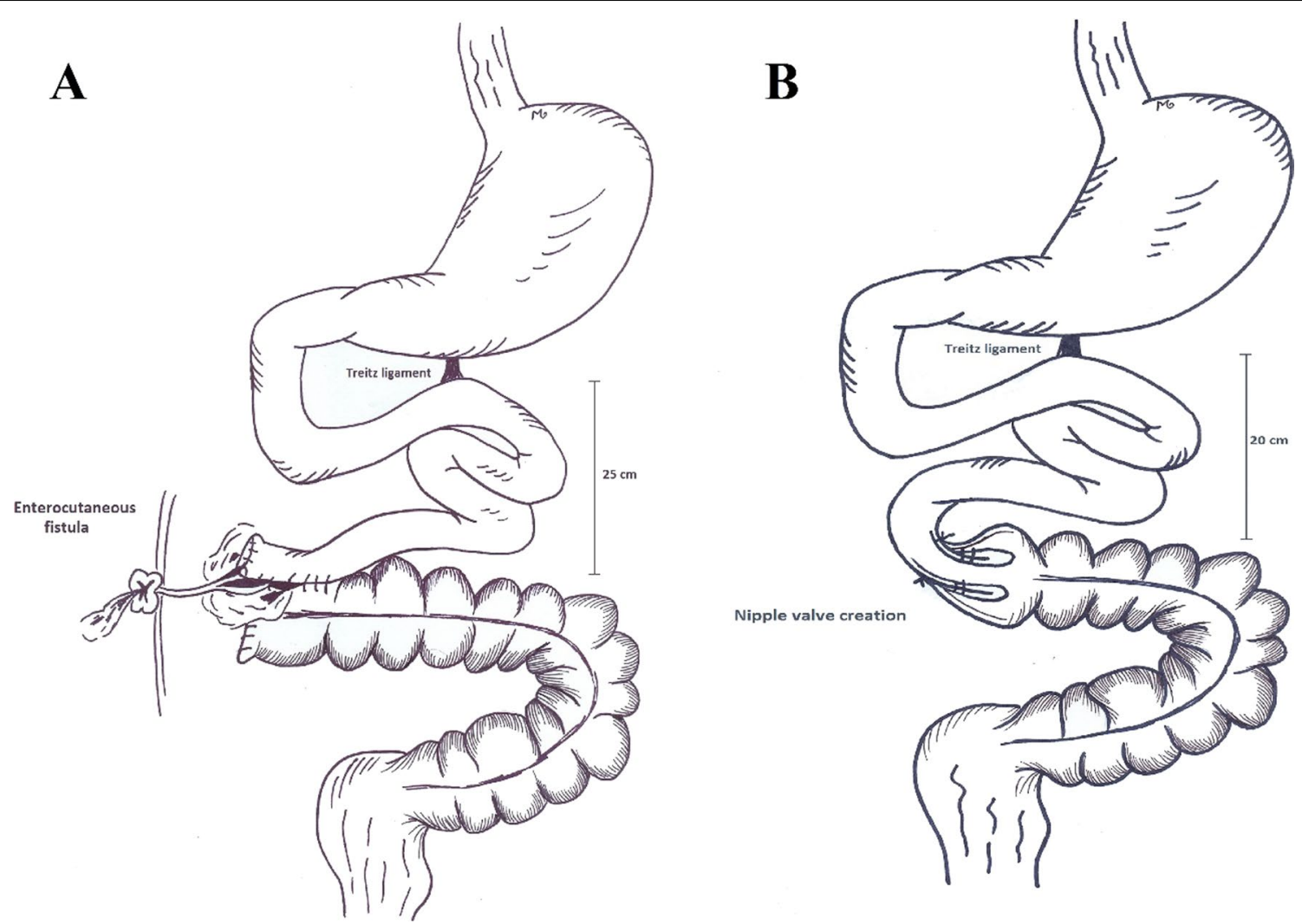

Fig. 4 The patient was transferred to our center after resection of a considerable length of bowel and with an enterocutaneous fistula (A). After resection, we created a nipple valve for the patient (B)

\section{Outcome}

The patient received $\mathrm{PN}$ for 4 weeks following surgery and was dismissed from the hospital at the end of the first post-operation month. 4 months after discharge he was once admitted with diarrhea and bacterial overgrowth which was managed by antibiotics. In the most recent follow up, after 10 months of surgery she had a satisfying quality of life without any symptoms regarding IF.

\section{Discussion and conclusion}

SBS is a debilitating condition with considerable disturbance of patients' quality of life and challenging management. Multidisciplinary teams are responsible to design a management plan for patients and to facilitate the small intestinal adaptation process. Once the small intestine fails, transplantation may be indicated. Considering the shortage of organs and transplantation risks, the team seeks solutions to avoid transplantation. In experienced hands, surgical techniques to lengthen the small intestinal absorptive mucosa are promising in alleviating the SBS condition [18]. To date, several small intestinal lengthening procedures have been invented and practiced worldwide. In 1980, Dr. Bianchi introduced the longitudinal intestinal lengthening and tailoring (LILT) procedure in pigs [19]. The technique was later widely practiced for SBS patients, particularly in the pediatric field. During the LILT procedure, the surgeon divides the intestine into two longitudinal halves, sutures the longitudinal sides and then reconnects the created segments to lengthen the intestine to two times as the primary length. Consequently, the final intestinal diameter is reduced to half [20]. In 2003, serial transverse enteroplasty (STEP) was introduced as a simpler method to achieve bowel lengthening by creating zigzag patterns in dilated bowel loops [14]. Since then, LILT and STEP have both been applied in IF centers with promising results. In a systematic review by Frongeia et al., both techniques were concluded to be as effective in intestinal lengthening and gaining relief of IF complications. However, STEP tends to have a slightly lower mortality rate and progression to transplantation [21]. In 2019, Shah et al., reported their 10-year experience in surgical management of SBS. The overall outcome of both techniques were similar in their follow up [22]. However debated, still the choice of the procedure mainly depends on surgeon's experience. 

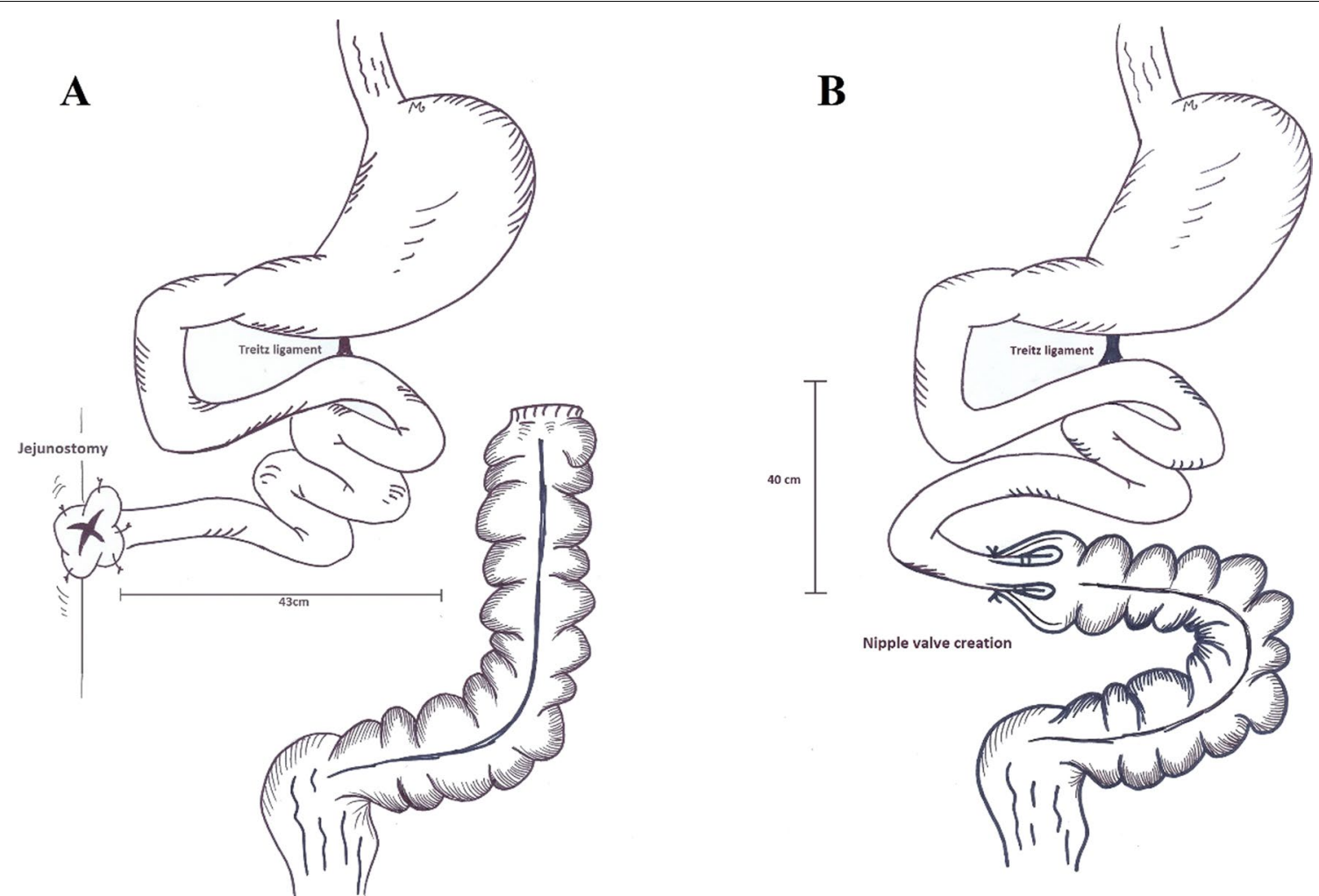

Fig. 5 The patient was referred after extensive resection of gangrened bowel and creation of a jejunostomy $(\mathbf{A})$. During AGIR, a nipple valve was created (B)

In our center, we are more experienced with STEP, as a less complicated procedure. Concerning of future stricture formation after LILT with our previous experiences, we prefer to perform STEP for patients with dilated bowel loops.

In this report, first we presented a case of considerable bowel shortness after resection due to mesenteric ischemia, who later underwent STEP procedure to lengthen the remaining intestine. The patient had initially underwent an exploratory laparotomy, fearing of short length of bowel remnants, the surgeon had decided to take no action. We suppose that with advances in AGIR techniques, hesitating to resect the gangrened bowel segments and delaying the procedure was an irrational choice. As, delayed resection of gangrened bowel segments is associated with higher mortality rate [23]. In presence of bowel gangrene, anastomoses will more frequently be accompanied by complications such as anastomosis leakage and fistula formation which contribute to further bowel shortening and consequent burdensome AGIR. Additionally, creating ostomies will bring about skin irritation and an additional bowel length loss after their closure [24]. Hence, we recommend closing the bowel ends and waiting for adaptive bowel dilatation.
A gastroduodenostomy tube is a desirable choice for decompression of gastric secretions [25]. In this case, we observed both lengthening and dilatation of the bowel remnants during the adaptive period. When in contrast study the dilated bowel segments had reached $4 \mathrm{~cm}$ in diameter, the patient was scheduled for STEP procedure. The gastroduodenotomy tube was removed 1 month later.

The ileocecal valve (ICV) prolongs the intestinal transit time, allowing more time for absorption. Besides, the valve prevents bacterial backwash from colon to ileum. The absence of ICV might result in ileal bacterial overgrowth and exacerbation of existing malabsorption [26]. Therefore, preserving the ICV in cases of small bowel resection helps reduce complications of SBS [27]. Furthermore, the duration of $\mathrm{PN}$ dependence is associated with remaining small bowel length and presence of ICV [28]. Absence of ICV also increases the need for repeated STEP [29]. The second presented case, discusses a patient with extremely short bowel remnant after resection of gangrened segments, who despite undergoing STEP needed an intestinal transplantation due to several admissions with dehydration and electrolyte imbalance. In cases of SBS, recurrent dehydration episodes, 
developing PN associated liver injury and repeated central line infection and thrombosis are among the indications of small intestinal transplantation [30]. For patients with viable bowel measuring less than $40 \mathrm{~cm}$ and loss of ileocecal valve, we recommend considering small intestine transplantation as the ultimate treatment strategy. Even additional duodenal lengthening via STEP procedure could not save the 2nd presented patient from repeated dehydration episodes. Further bowel lengthening techniques including LILT was considered for the patient, however little chance of living a PN-free life was expected in a condition with extreme short length of bowel. Meanwhile she was put on transplantation waiting list as a treatment strategy to finalize patient's discharge from hospital. Complications of $\mathrm{PN}$, repeated admissions due to dehydration and delayed bowel transplantation adversely affected the transplantation outcome. In our country, home PN is not practiced; therefore, we recommend intestinal transplantation as the primary management strategy for patients with extremely SBS.

In the 3rd case, we presented a liver recipient lady with Roux-en-Y reconstruction who lost a considerable length of her intestines due to internal herniation and consequent gangrene. In assessment of abdominal pain in patients who have undergone Roux-en-Y reconstruction, possibility of internal herniation should always be considered and if indicated, patients should immediately undergo exploratory laparotomy to prevent bowel gangrene [31]. Approach to abdominal pain in organ recipients is not considerably different from normal population. Therefore, in cases of acute abdomen, emergent laparotomies should not be postponed until the patients are referred to organ transplantation centers [32]. In the absence of peritonitis and evidences of abdominal contamination; while bowel loops were dilated, we performed an early STEP to regain intestinal autonomy. We avoided anastomosis creation in the first surgery, considering the fact that the patient was unstable and immunosuppressed. Since the immunosuppressant agents are absorbed in duodenum and jejunum, we inserted a gastroduodenostomy tube to provide a safe route for medications. With the aid of Cattell-Braasch maneuver, a tension free biliary anastomosis was provided.

The 4th case discusses a 72-year-old patient who had underwent immediate resection of gangrened bowel segments and anastomosis of the viable tissue due to mesenteric ischemia. As earlier mentioned, in cases of bowel gangrene primary anastomosis might be complicated by anastomosis leakage and fistula, as what this patient experienced. With the lack of ileocecal valve and the viable bowel measuring $20 \mathrm{~cm}$, small intestine transplantation was the optimal treatment strategy. However, considering patient's age and performance status, he was not an appropriate candidate for transplantation. By creating a nipple valve, the patient was less frequently dependent on PN and his quality of life appreciably improved. It seemed that the nipple valve helped the patient to be discharged from hospital with an extremely short length of small bowel. Therefore, the method of AGIR differs based on patient's performance status.

In the 5th case, we presented a lady with a stoma and closure of transverse colon after primary resection and anastomosis. Considering the fact that most patients are referred to IRUs after primary operations, the MDT should be prepared for intestinal reconstruction in various conditions, namely in presence of stomas. For patients with stomas, regular diet is started as soon as possible and an expert nurse cares for the stoma. Besides, the patient received PN for 8 weeks after the primary operation. During the intestinal reconstruction operation, $43 \mathrm{~cm}$ of bowel was remaining and the ileocecal valve was lost; therefore, we decided to create a nipple valve by everting $3 \mathrm{~cm}$ of jejunum and telescoping the its tip to the colon. As the final step, the base of the nipple was anastomosed to colon. A gastroduodenostomy tube was inserted as a vent. The patient was transferred to IRU and as soon as he could obtain $60 \%$ of his daily calorie form oral nutrition, PN was tapered. With no nipple valve creation, the patient would have been a candidate for small intestine transplantation. In our center, we have adopted the method of nipple valve for patients with $40-80 \mathrm{~cm}$ bowel remnant with concurrent ileocecal valve loss and no bowel dilatation.

In the current manuscript we reviewed 5 cases of SBS as a consequence of laparotomies. Adopting the STEP procedures helped 2 patients to be gradually weaned from $\mathrm{PN}$; however one patient with extremely short bowel length and ICV absence did not benefit from the procedure. Nipple valve reconstruction was approached for 2 patients lacking ICV, with acceptable surgery outcome.

\section{Abbreviations}

AGIR: Autologous gastrointestinal reconstruction; ICV: Ileocecal valve; IRU: Intestinal rehabilitation unit; LILT: Longitudinal intestinal lengthening and tailoring; NPO: Nil per Os; OR: Operation room; PN: Parenteral nutrition; STEP: Serial transverse enteroplasty; SBS: Short bowel syndrome.

\section{Acknowledgements}

Not applicable.

\section{Authors' contributions}

HN was the chief surgeon to attend the patients. KA was the hepatobiliary fellow who attended the surgeries and was in charge of following patients. MSH is the clinical pharmacist to design PN in the IRU. HN wrote the reports. $\mathrm{KA}, \mathrm{MSH}, \mathrm{ME}$ and NA assisted in preparation of the manuscript. ME drew the illustrations. All authors reviewed the paper and agreed to be accountable for all aspects of the work in ensuring that questions related to the accuracy or integrity of any part of the work are appropriately investigated and resolved. All authors read and approved the final manuscript. 


\section{Funding}

No funding was applied for this study.

\section{Availability of data and materials}

All data generated or analysed during this study are included in this published article.

\section{Declarations}

\section{Ethics approval and consent to participate}

The study involving a human participant to report was approved by the ethics committee of the department of Transplantation at Shiraz University of Medical Sciences. Written consent was obtained from the patients.

\section{Consent for publication}

Written informed consent was obtained from the all the patients or next of kin in regarding deceased patients, for publication of their personal and clinical details along with any identifying images to be published in the study. A copy of the written consent is available for review by the Editor of this journal.

\section{Competing interests}

The authors declare no competing interests.

\section{Author details}

${ }^{1}$ Shiraz Transplant Research Center, Shiraz University of Medical Sciences, Shiraz, Iran. ${ }^{2}$ Department of Clinical Pharmacy, Faculty of Pharmacy, Shiraz University of Medical Sciences, Shiraz, Iran.

Received: 19 August 2021 Accepted: 24 December 2021

Published online: 30 December 2021

\section{References}

1. Pironi L, Arends J, Baxter J, Bozzetti F, Peláez RB, Cuerda C, et al. ESPEN endorsed recommendations. Definition and classification of intestinal failure in adults. Clin Nutr. 2015;34(2):171-80.

2. Jeppesen PB. Spectrum of Short Bowel Syndrome in Adults. J Parenter Enter Nutr. 2014;38(1S):8S-13S.

3. Buchman AL, Scolapio J, Fryer J. AGA technical review on short bowel syndrome and intestinal transplantation. Gastroenterology. 2003:124(4):1111-34.

4. Nightingale JMD. Hepatobiliary, renal and bone complications of intestinal failure. Best Pract Res Clin Gastroenterol. 2003;17(6):907-29.

5. Welters CFM, Dejong CHC, Deutz NEP, Heineman E. Intestinal adaptation in short bowel syndrome. ANZ J Surg. 2002;72(3):229-36.

6. Sondheimer JM, Cadnapaphornchai M, Sontag M, Zerbe GO. Predicting the duration of dependence on parenteral nutrition after neonatal intestinal resection. J Pediatr. 1998;132(1):80-4.

7. Buchman AL. Etiology and initial management of short bowel syndrome. Gastroenterology. 2006;130(2):S5-15.

8. Jones BA, Hull MA, McGuire MM, Kim HB. Autologous intestinal reconstruction surgery. Semin Pediatr Surg. 2010. https://doi.org/10.1053/j. sempedsurg.2009.11.007.

9. Allan P, Lal S. Intestinal failure: a review. F1000Res. 2018;7:85.

10. Ashrafzadeh K, Shafiekhani M, Azadeh N, Esmaeili M, Nikoupour H. Lessons learned from successful autologous gastrointestinal reconstruction in patients with intestinal failure: a case series. BMC Surg. 2021;21(1):73.

11 Morabito A, Ugolini S, Cianci MC, Coletta R. Current surgical concepts and indications in the management of the short bowel state: a call for the use of multidisciplinary intestinal rehabilitation programs. Children (Basel, Switzerland). 2021. https://doi.org/10.3390/children8080654.

12 Nikoupour H, Arasteh P, Shamsaeefar A, Ghanbari F, Boorboor A, Almayali AMJ, et al. Experiences with intestinal failure from an intestinal rehabilitation unit in a country without home parenteral nutrition. J Parenter Enter Nutr. 2021. https://doi.org/10.1002/jpen.2231.

13. Ramos-Gonzalez G, Kim HB. Autologous intestinal reconstruction surgery. Semin Pediatr Surg. 2018:27(4):261-6.
14. Kim HB, Fauza D, Garza J, Oh J-T, Nurko S, Jaksic T Serial transverse enteroplasty (STEP): a novel bowel lengthening procedure. J Pediatr Surg. 2003:38(3):425-9.

15. Barrett M, Demehri FR, Ives GC, Schaedig K, Arnold MA, Teitelbaum DH. Taking a STEP back: assessing the outcomes of multiple STEP procedures. J Pediatr Surg. 2017;52(1):69-73

16. Mercer DF, Hobson BD, Gerhardt BK, Grant WJ, Vargas LM, Langnas AN et al. Serial transverse enteroplasty allows children with short bowel to wean from parenteral nutrition. J Pediatr. 2014;164(1):93-8.

17. Barksdale EM, Stanford A. The surgical management of short bowel syndrome. Curr Gastroenterol Rep. 2002;4(3):229-37.

18. Donohoe CL, Reynolds JV. Short bowel syndrome. Surgeon. 2010;8(5):270-9.

19. Bianchi A. Intestinal loop lengthening - a technique for increasing small intestinal length. J Pediatr Surg. 1980;15(2):145-51.

20. Chahine AA, Ricketts RR. A modification of the Bianchi intestinal lengthening procedure with a single anastomosis. J Pediatr Surg. 1998;33(8):1292-3.

21. Frongia G, Kessler M, Weih S, Nickkholgh A, Mehrabi A, Holland-Cunz S. Comparison of LILT and STEP procedures in children with short bowel syndrome - a systematic review of the literature. J Pediatr Surg. 2013;48(8):1794-805

22. Shah AA, Petrosyan M, Franklin AL, Chahine AA, Torres C, Sandler AD. Autologous intestinal reconstruction: a single institution study of the serial transverse enteroplasty (STEP) and the longitudinal intestinal lengthening and tailoring (LILT). Pediatr Surg Int. 2019;35(6):649-55

23. Wadman M, Syk I, ElmståhI S. Survival after operations for ischaemic bowel disease. Eur J Surg. 2000;166(11):872-7.

24. Kwiatt M, Kawata M. Avoidance and management of stomal complications. Clin Colon Rectal Surg. 2013;26(2):112-21.

25. Cruz RJ Jr, Butera L, Poloyac K, McGurgan J, Stein W, Binion D, et al. Surgical and medical approach to patients requiring total small bowel resection: managing the "no gut syndrome." Surgery. 2017;162(4):871-9.

26. Ziegler MM. Short bowel syndrome in infancy: etiology and management. Clin Perinatol. 1986;13(1):163-73.

27. Höllwarth ME. Surgical strategies in short bowel syndrome. Pediatr Surg Int. 2017;33(4):413-9.

28. Goulet O, Baglin-Gobet S, Talbotec C, Fourcade L, Colomb V, Sauvat F, et al. Outcome and long-term growth after extensive small bowel resection in the neonatal period: a survey of 87 children. Eur J Pediatr Surg. 2005;15(02):95-101.

29. Wester T, Lilja HE, Stenström P, Pakarinen M. Absent ileocecal valve predicts the need for repeated step in children. Surgery. 2017;161(3):818-22.

30. Abu-Elmagd KM. Intestinal transplantation for short bowel syndrome and gastrointestinal failure: current consensus, rewarding outcomes, and practical guidelines. Gastroenterology. 2006;130(2 Suppl 1):S132-7.

31. Elms L, Moon RC, Varnadore S, Teixeira AF, Jawad MA. Causes of small bowel obstruction after Roux-en-Y gastric bypass: a review of 2,395 cases at a single institution. Surg Endosc. 2014:28(5):1624-8.

32 Cesaretti M, Dioguardi Burgio M, Zarzavadjian Le Bian A. Abdominal emergencies after liver transplantation: presentation and surgical management. Clin Transplant. 2017;31(11):e13102.

\section{Publisher's Note}

Springer Nature remains neutral with regard to jurisdictional claims in published maps and institutional affiliations. 